RESEARCH ArTICLE

Published April 26, 2021

\title{
Alpha 1 Antitrypsin is an INHIBITOR OF THE SARS-COV-2-PRIMING ProteAse TMPRSS2
}

\section{AUTHORS}

Nurit P. Azouz ${ }^{1,2^{*}}$, Andrea M. Klingler ${ }^{1}$, Victoria Callahan ${ }^{3}$, Ivan V. Akhrymuk ${ }^{3,4}, K_{\text {Katarina Elez }}^{5}$, Lluís Raich ${ }^{5}$, Brandon M. Henry ${ }^{6}$, Justin L. Benoit ${ }^{7}$, Stefanie W. Benoit ${ }^{8,2}$, Frank Noés,9,10, Kylene Kehn-Hall ${ }^{3,4}$ and Marc E. Rothenberg ${ }^{1,2}$

\section{AFFILIATED INSTITUTIONS}

${ }^{1}$ Division of Allergy and Immunology, Cincinnati Children's Hospital Medical Center, Department of Pediatrics, University of Cincinnati College of Medicine, Cincinnati, OH. ${ }^{2}$ Department of Pediatrics, University of Cincinnati College of Medicine, Cincinnati, OH.

${ }^{3}$ National Center for Biodefense and Infectious Diseases, School of Systems Biology, George Mason University, Manassas, VA.

${ }^{4}$ Department of Biomedical Sciences and Pathobiology, Virginia Polytechnic Institute and State University, Blacksburg, VA.

${ }^{5}$ Freie Universität Berlin, Department of Mathematics and Computer Science, Berlin, Germany. ${ }^{6}$ Cardiac Intensive Care Unit, The Heart Institute, Cincinnati Children's Hospital Medical Center, Cincinnati, $\mathrm{OH}$.

${ }^{7}$ Department of Emergency Medicine, University of Cincinnati, Cincinnati, OH.

${ }^{8}$ Division of Nephrology and Hypertension, Cincinnati Children's Hospital Medical Center, Cincinnati, $\mathrm{OH}$.

${ }^{9}$ Freie Universität Berlin, Department of Physics, Berlin, Germany.

${ }^{10}$ Rice University, Department of Chemistry, Houston, TX.

CORRESPONDING AUTHOR DOI

Nurit P. Azouz, PhD

Cincinnati Children's Hospital Medical Center, Cincinnati, $\mathrm{OH}$

nurit.azouz@cchmc.org

ORCID: 0000-0003-4969-1263

\section{SUGGESTED CITATION}

Azouz NP, Klingler AM, Callahan V, Akhrymuk IV, Elez K, Raich L, Henry BM, Benoit JL, Benoit SW, Noé F, Kehn-Hall K, Rothenberg ME. Alpha 1 Antitrypsin is an Inhibitor of the SARS- 
CoV-2-Priming Protease TMPRSS2. Pathogens and Immunity. 2021;6(1):55-74. Doi:10.20411/ pai.v6i1.408

\section{ABSTRACT}

Background: Host proteases have been suggested to be crucial for dissemination of MERS, SARS$\mathrm{CoV}$, and SARS-CoV-2 coronaviruses, but the relative contribution of membrane versus intracellular proteases remains controversial. Transmembrane serine protease 2 (TMPRSS2) is regarded as one of the main proteases implicated in the coronavirus $S$ protein priming, an important step for binding of the $\mathrm{S}$ protein to the angiotensin-converting enzyme 2 (ACE2) receptor before cell entry.

Methods: We developed a cell-based assay to identify TMPRSS2 inhibitors. Inhibitory activity was established in SARS-CoV-2 viral load systems.

Results: We identified the human extracellular serine protease inhibitor (serpin) alpha 1 antitrypsin (A1AT) as a novel TMPRSS2 inhibitor. Structural modeling revealed that A1AT docked to an extracellular domain of TMPRSS2 in a conformation that is suitable for catalysis, resembling similar serine protease inhibitor complexes. Inhibitory activity of A1AT was established in a SARS-CoV-2 viral load system. Notably, plasma A1AT levels were associated with COVID-19 disease severity.

Conclusions: Our data support the key role of extracellular serine proteases in SARS CoV-2 infections and indicate that treatment with serpins, particularly the FDA-approved drug A1AT, may be effective in limiting SARS-CoV-2 dissemination by affecting the surface of the host cells.

Keywords: COVID; coronavirus; TMPRSS2; protease; alpha 1 antitrypsin; camostat mesylate

\section{INTRODUCTION}

The COVID-19 pandemic is caused by the severe acute respiratory syndrome (SARS)-coronavirus $(\mathrm{CoV})-2$. The efficient transmission of this virus has led to exponential growth in the number of worldwide cases. Similar to other coronaviruses, SARS-CoV-2 entry into host cells relies on the proteolytic processing of spike (S) protein by host proteases and engagement of the angiotensin-converting enzyme 2 (ACE2) receptor [1]. Several proteases are crucial to coronavirus viral entry, and they are found at different subcellular locations. The $\mathrm{S}$ protein cleavage may occur extracellularly near the plasma membrane by cell surface proteases or intracellularly by lysosomal endopeptidase enzymes, such as cathepsin L, which facilitate viral entry by activating membrane fusion and subsequent cell entry through endocytosis, as in the case of MERS-CoV [2] . Despite intensive research of the SARS-CoV-2 life cycle, the cellular location of the SARS-CoV-2 S protein priming remains debatable. In particular, the interplay between the extracellular and intracellular proteases in the membrane fusion and cell entry of SARS-CoV-2 is controversial.

Transmembrane serine protease 2 (TMPRSS2), a cell surface serine protease, may be involved in cell entry of SARS-CoV-2. TMPRSS2 has been shown to cleave ACE2 at arginine and lysine residues within ACE2 amino acids 697-716, which enhances cell entry [ $\underline{3}$ ]. TMPRSS2 increases the entry of another coronavirus SARS-CoV, not only by processing of the S protein, but also by processing of the host receptor ACE2 [3] . Consistent with these findings, TMPRSS2-deficient 
mice have decreased viral spread of SARS-CoV in the airways compared to that of control mice [4]. In addition, the drug camostat mesylate (camostat), which inhibits a number of proteases including TMPRSS2, was shown to inhibit SARS-CoV-2 entry into cells in vitro $[\underline{1}, \underline{5}]$. Therefore, TMPRSS2 is regarded as one of the most important proteases for S protein priming and cell entry of SARS-CoV-2.

Herein, we developed a cell-based assay to identify TMPRSS2 inhibitors. We compared the efficiency of TMPRSS2 inhibition by synthetic and natural serine protease inhibitors that are cell permeable or have extracellular function, including drugs with known function as protease inhibitors. We identified alpha 1 antitrypsin (A1AT) as a novel inhibitor of TMPRSS2. Structural modeling of the Michaelis complex between TMPRSS2 and A1AT indicated that they dock on the cell surface in a conformation that is suitable for catalysis, resembling similar serine protease-inhibitor complexes. We further provided proof of concept for the potential utility of A1AT as an antiviral agent in SARS-CoV-2 infection. A1AT decreased SARS-CoV-2 copy number within target cells when applied during infection. The effect of A1AT was comparable to the effect of camostat, which was previously shown to inhibit SARS-CoV-2 cell entry [1]. In contrast to camostat, which is a cell permeable drug [] ], A1AT is a circulating extracellular protein that inhibits extracellular proteases and does not possess access to intracellular proteases [7]. These findings emphasize the importance of extracellular proteases to viral cell entry. We suggest that by inhibiting extracellular proteolytic activity, A1AT can potentially inhibit S protein processing and limit SARS-CoV-2 cellcell spread and dissemination.

\section{METHODS}

\section{Materials}

Secretory leukocyte peptidase inhibitor (SLPI) and Boc-Gln-Ala-Arg-7-Amino-4-methylcoumarin (BOC-QAR-AMC) were obtained from R\&D systems. Camostat mesylate was obtained from Sigma Aldrich, and A1AT (CSL Behring, Zemaira and Grifols, Prolastin-C) was a kind gift of Mark Brantly (University of Florida, Gainesville, FL). Recombinant TMPRSS2 was obtained from Abnova.

\section{TMPRSS2 overexpression}

A PLX304 plasmid-containing human TMPRSS2 open reading frame from the ORFeome Collaboration (Dana-Farber Cancer Institute, Broad Institute of Harvard and Massachusetts Institute of Technology [HsCD00435929]) was obtained from DNASU Plasmid Repository, and a control PLX304 vector was obtained from Addgene (Watertown, MA, USA).

\section{HEK-293T cell culture and transfection}

HEK-293T cells were grown in Dulbecco's Modified Eagle Medium (DMEM) supplemented with $10 \%$ fetal bovine serum (FBS) and seeded in a black, 96-well plate (75,000 cells/well). The following day, cells were transfected overnight with either a control plasmid (PLX) or TMPRSS2 (PLX-TMPRSS2) using TransIT LT-1 transfection reagent (Mirus Bio) in $100 \mu \mathrm{L}$ of OptiMEM per well. The media was replaced the day following the overnight transfection. 


\section{TMPRSS2 activity assay}

Twenty-four hours after transfection, the media was replaced with $80 \mu \mathrm{L}$ of phosphate-buffered saline (PBS). Inhibitors or PBS alone were added to the wells in the indicated concentrations and incubated at $25^{\circ} \mathrm{C}$ for 15 minutes. The fluorogenic substrate BOC-QAR-AMC (R\&D Biosystems) was then added to each well to a final concentration of $100 \mu \mathrm{M}$. Fluorescence (excitation $365 \mathrm{~nm}$, emission $410 \mathrm{~nm}$ ) was immediately measured every 15 minutes at $37^{\circ} \mathrm{C}$ using a GloMax plate reader (Promega).

\section{Gel electrophoresis and western blot}

Protein lysates of HEK-293T cells were extracted with RIPA buffer (PIERCE) and protease inhibitor cocktail (Roche). Loading buffer (Life Technologies) was added, and samples were heated to $95^{\circ} \mathrm{C}$ for 5 minutes and subjected to electrophoresis in 12\% NuPAGE Bis-Tris gels (Life Technologies). Gels were transferred to nitrocellulose membranes (Life Technologies) and probed with the primary antibodies rabbit anti-V5 (Bethyl Laboratories), mouse anti-TMPRSS2 (Santa Cruz), and rabbit anti-human GAPDH (ABCAM) and subsequently with the secondary antibody IRDye 800RD goat anti-rabbit and IRDye 680RD goat anti-mouse (LI-COR Biosciences). Membranes were visualized and analyzed using the Odyssey CLx system (LI-COR Biosciences). For TMPRSS2 quantity estimations, recombinant TMPRSS2 was subjected to gel electrophoresis with protein lysates from TMPRSS2-overexpressing cells.

\section{Cell viability assay}

HEK-293T cells were grown for 48 hours in 96-well plates. Then cells were treated with the indicated concentrations of A1AT for an additional 3.25 hours. Cell viability was assessed by Alamar blue cell viability reagent (ThermoFisher) according to the manufacturer's protocol.

Caco-2 cells were plated in 96-well plates. Cells were treated with A1AT or camostat at the indicated concentrations for 18 hours. Cell viability was estimated by CellTiter-Glo Cell Viability Assay from Promega according to the manufacturer's protocol.

\section{Quantifying SARS-CoV-2 genomic copies}

Caco-2 cells were plated in 12-well plates and pretreated for 1 hour prior to SARS-CoV-2 infection with DMEM containing A1AT or camostat in the indicated concentrations or DMEM alone (media-only control). Cells were incubated with SARS-CoV-2 (through BEI Resources, NIAID, NIH: SARS-Related Coronavirus 2 Isolate USA-WA1/2020, NR-52281) at a multiplicity of infection of 0.1 and with the indicated drugs for 1 hour. Then, cells were washed with DMEM and incubated with DMEM containing A1AT or camostat or DMEM alone. Twenty hours post infection, RNA was extracted from the cells, and viral copy number was quantified by real-time, quantitative PCR.

\section{mRNA extraction and real-time, quantitative PCR}

Cells were lysed with TriZol LS, and total RNA was isolated from cells with the Direct-zol RNA mini prep kit (Zymo research) according to the manufacturer's protocol. RNA was treated with the On-Column DNase Digestion kit (Qiagen) according to the supplied protocol. The amount of the intracellular RNA was estimated by real-time quantitative PCR performed with the RNA 
UltraSense $^{\mathrm{Tm}}$ One-Step Quantitative RT-PCR System (Applied Biosystems) by using the following primer sets: CDC N1 (forward 5'- GAC CCC AAA ATC AGC GAA AT, reverse 5'- TCT GGT TAC TGC CAG TTG AAT CTG, probe 5'-FAM- ACC CCG CAT TAC GTT TGG TGG ACC -BHQ1), 18S rRNA (TaqMan Gene expression assay from ThermoFisher, Hs99999901_s1), and GusB (TaqMan Gene expression assay from ThermoFisher, Hs99999908_m1).

\section{SARS-CoV-2 plaque assay}

Virus concentration was estimated by traditional plaque assay as previously described []. Briefly, Calu-3 cells were plated in a 12 -well plate at $2 \times 10^{5}$ cell density and incubated overnight. The cell monolayer was infected by serially diluted virus and covered with $0.6 \%$ agarose. Cells were fixed with $10 \%$ paraformaldehyde 48 hours post infection. Crystal violet staining was used for plaque visualization and plaque counting.

\section{Template-based molecular docking}

The Michaelis complex of TMPRSS2 and A1AT was modeled using HADDOCK v2.4 [9] , a versatile docking engine guided by structural data from experiments and/or computational models. A complex between S195A bovine cationic trypsin and the Pittsburgh variant of A1AT (PDB 1OPH [10]) was taken as a template to drive the docking process, as well as to obtain the structure of A1AT (chain A, reverted to its wild-type form). The structure of TMPRSS2 was obtained from reference [11] (homology model based on PDB 3W94). PS-HomPPI v2.0 [12] was used for mapping the interface residues of PDB 1OPH onto the query structures and calculating their CA-CA distances. A subset of these distances within different cutoffs $(7,9,10,11$, and $15 \AA$ ) was then used as unambiguous restraints to drive the docking between TMPRSS2 and A1AT. The number of structures generated in each of the docking phases, ie, rigid-body, semi-flexible, and water refinement, was kept at 1000, 200, and 200, respectively. The top 4 models generated using each of the 5 cutoffs were analyzed, and the final model was selected on the basis of visual inspection and energetic terms.

\section{COVID-19 plasma analysis}

Adults who presented to the University of Cincinnati Medical Center (UCMC) Emergency Department (ED) with suspected COVID-19 and had a clinically indicated blood draw were prospectively enrolled via an institutional review board-approved waiver of informed consent. Following collection, samples were centrifuged at $2000 \mathrm{~g}$ for 15 minutes at $4^{\circ} \mathrm{C}$ and frozen at $-80^{\circ} \mathrm{C}$ until analysis. Inclusion in this analysis was dependent on a positive reverse transcription polymerase chain reaction (RT-PCR) test for COVID-19 via a standard-of-care nasopharyngeal swab. A total of 49 patients were included. The median age was 46.5 years (IQR: $37-66$ ). The plasma concentration of A1AT was measured on a Behring Nephelometer II System (BN II, Siemens Medical Solutions USA, Inc., Malvern, PA, USA). The plasma concentrations of IL-6, IL-8, IL-10, and TNFa were quantified using the Meso Scale Discovery (MSD) U-Plex assay (Rockville, Maryland, USA). Patients who were positive for COVID-19 were stratified into subgroups on the basis of disease severity at ED disposition and maximal disease severity within 30 days of the index ED visit. Mild COVID-19 was defined as illness requiring only outpatient care (level 1), moderate COVID-19 was defined as illness requiring hospitalization (level 2), and severe COVID-19 was defined as illness requiring Intensive Care Unit admission and/or mechanical ventilation and/or resulting in the death of the patient. Comparisons of plasma concentrations of A1AT with those 
of IL-10, IL-6, IL-8, and TNFa were performed using the Spearman's test.

\section{Statistics}

$\mathrm{IC}_{50}$ values were calculated by nonlinear regression by dose-response inhibition with variable slopes. Statistical significance was determined using a $t$ test (unpaired, 2-tailed). Statistical analyses were performed using GraphPad Prism (GraphPad Software Incorporated).

\section{RESULTS}

\section{Overexpressing TMPRSS2 and measuring proteolytic activity}

We aimed to establish an experimental framework for quantifying TMPRSS2 proteolytic activity. We chose to overexpress TMPRSS2 with a C-terminal V5 tag, in a human cell line, HEK-293T, because of its high transfectability. Western blot analysis of the cell lysates revealed a band at $\sim 60$ $\mathrm{kD}$ in TMPRSS2-transfected cells but not in control cells (Figure 1A). GAPDH was used as a loading control. Measurements of the proteolytic activity of the transfected cells using the fluorogenic peptide substrate Boc-Gln-Ala-Arg-7-Amino-4-methylcoumarin (BOC-QAR-AMC) revealed a $>2.5$-fold increase in the proteolytic activity of the TMPRSS2-transfected cells compared with that of control cells $(P=0.0002$; Figure $1 \mathrm{~B})$. The proteolytic activity of the TMPRSS2-transfected cells was increased compared with that of control cells (Figure 1C). The mean proteolytic rate per minute of the TMPRSS2-transfected cells was increased by $>3.5$ fold compared to the proteolytic rate of control cells $(P<0.0001$, Figure 1D). Using serial dilutions of recombinant TMPRSS2, we estimated that the amount of TMPRSS2 that is expressed by TMPRSS2-overexpressing cells is about $100 \mathrm{ng} /$ well (Supplementary Figure 1). These collective data demonstrated that overexpression of TMPRSS2 resulted in overproduction of functional TMPRSS2 and established an experimental system for accurately measuring the proteolytic activity of TMPRSS2.

\section{Identifying functional TMPRSS2 inhibitors}

We tested the effect of protease inhibitors on TMPRSS2 activity. As a positive control, cells were treated with camostat mesylate, a drug that has been shown to inhibit TMPRSS2 $[\underline{1}, \underline{13}]$. As expected, camostat mesylate inhibited the proteolytic activity of TMPRSS2 with a calculated $\mathrm{IC}_{50}$ of $42 \mathrm{nM}$ (Figure 2A). We then tested whether the secretory leukocyte protease inhibitor (SLPI) would inhibit TMPRSS2. However, none of the tested concentrations of SLPI inhibited TMPRSS2 proteolytic activity (Figure 2B). In contrast, A1AT inhibited TMPRSS2 proteolytic activity in a dose-dependent manner ( $\mathrm{IC}_{50}$ of $357 \mathrm{nM}$; Figure $2 \mathrm{C}$ ). A1AT did not demonstrate toxic effects in the tested concentrations as demonstrated by viability assays of HEK-293T cells (Supplementary Figure 2).

\section{Modeling the extracellular TMPRSS2-A1AT Michaelis complex}

We modeled the Michaelis complex between TMPRSS2 and A1AT to better understand the structural basis of TMPRSS2 inhibition prior to A1AT cleavage and covalent attachment (Figure 3A). Our results suggest that TMPRSS2 interacts with A1AT through its reactive center loop (RCL), driven by complementary electrostatic interactions at their surfaces (Figure 3B). Namely, LYS390 (TMPRSS2) forms a strong bifurcated salt bridge with GLU199/ASP202 (A1AT), while LYS340 and ASP260 form a second electrostatic contact, whose proximity may be limited by the presence of ASP338 at the surface of TMPRSS2. In the active site region, TMPRSS2 interacts with A1AT via 
an extensive hydrogen bond network (Figure 3C). Part of these interactions stabilizes a short, antiparallel beta-sheet between GLY462 and ILE356-PRO357, similar to the one present in 1 OPH of the Research Collaboratory for Structural Bioinformatics (RCSB) Protein Data Bank (PDB) [10]. At the entrance of the S1 pocket, GLN438 forms hydrogen bonds with PRO357 and SER359, fixing and orienting the A1AT backbone around the reactive peptide bond (MET358-SER359). As a result, MET358 is buried inside the S1 pocket, with its backbone carbonyl placed inside the oxyanion hole, forming hydrogen bonds with the GLY439 and SER441 (TMPRSS2) backbone amides. These predicted interactions, together with the overall orientation of the RCL and the catalytic triad, constitute a suitable environment for the cleavage and covalent binding of A1AT to TMPRSS2.

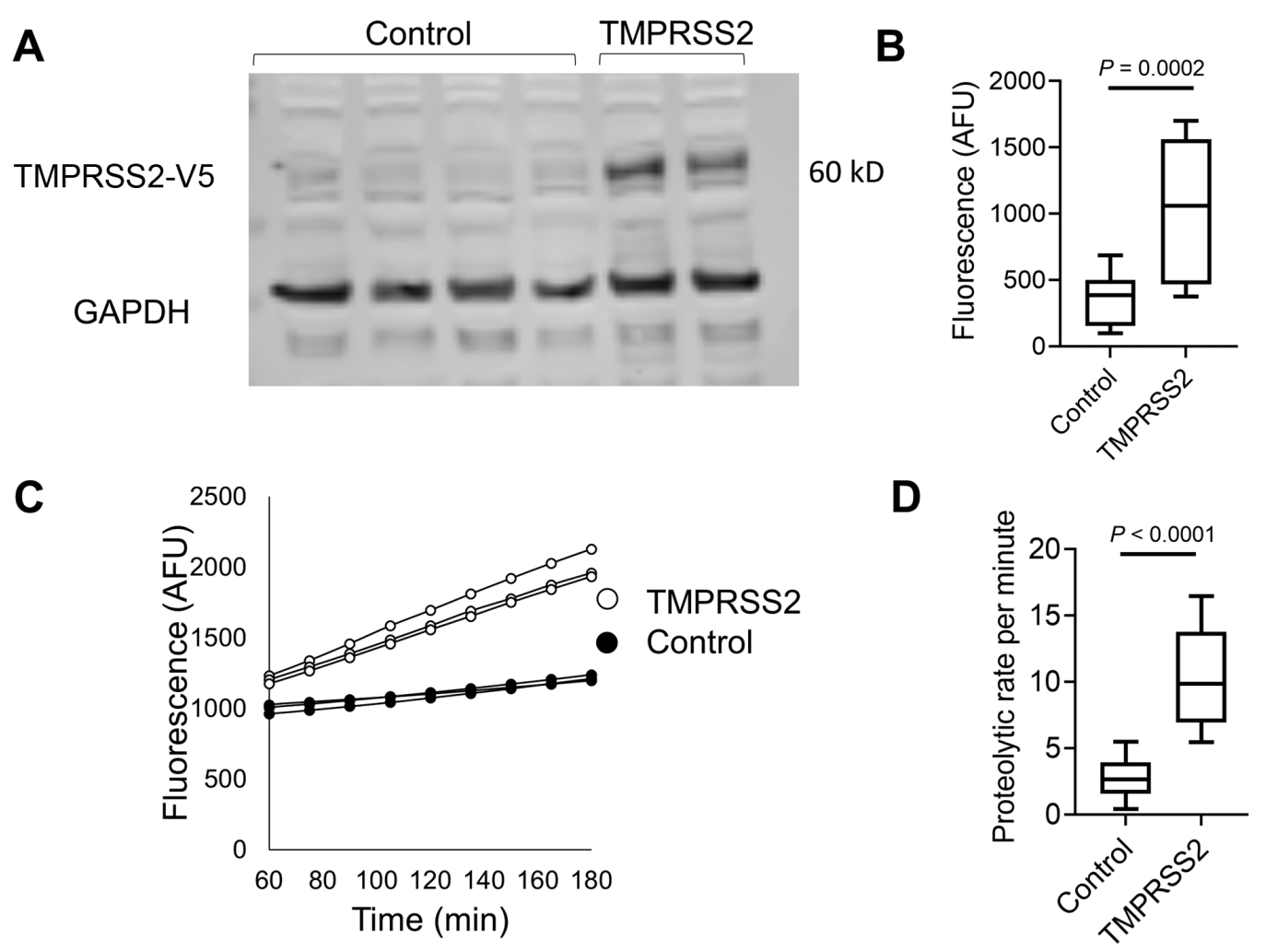

Figure 1. Measurements of TMPRSS2 activity in transfected cells.

A. Western blot of TMPRSS2 protein expression in HEK-293T cells transfected with PLX304 vector or PLX304-TMPRSS2 vector is shown. TMPRSS2 containing a C terminal V5 (TMPRSS2-V5) tag was assessed by anti-V5 antibody, and anti-GAPDH antibody was used as a loading control. B. Arbitrary fluorescence unit (AFU) measurements of control or TMPRSS-overexpressing cells incubated with BOCQAR-AMC for 75 minutes at $37^{\circ} \mathrm{C}$ are shown. Wells containing PBS and BOC-QAR-AMC were used as background fluorescence reads. C. Fluorescence of control or TMPRSS2-overexpressing cells was measured every 15 minutes for a total time of 180 minutes. D. The average proteolytic activity rate per minute of control or TMPRSS2-overexpressing cells. The fluorescent signal was measured by the UV filter (excitation $365 \mathrm{~nm}$ and emission $410 \mathrm{~nm}$ ). Data in B and D represent the mean \pm SD with interquartile ranges. GAPDH, glyceraldehyde-3-phosphate dehydrogenase; TMPRSS2, transmembrane serine protease 2. 
A

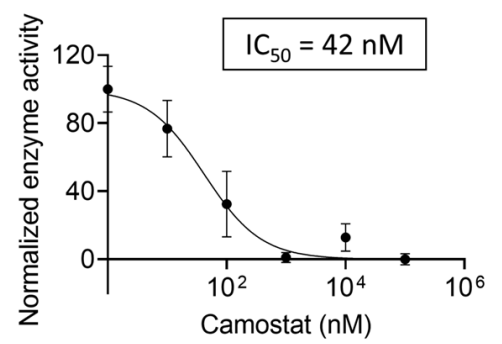

B

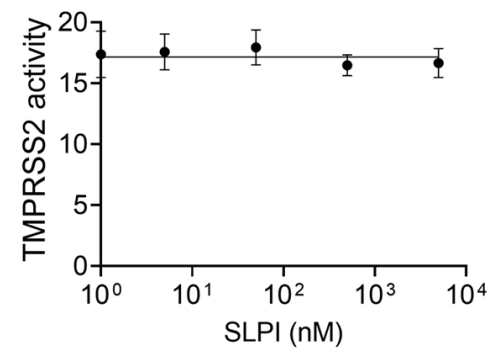

C

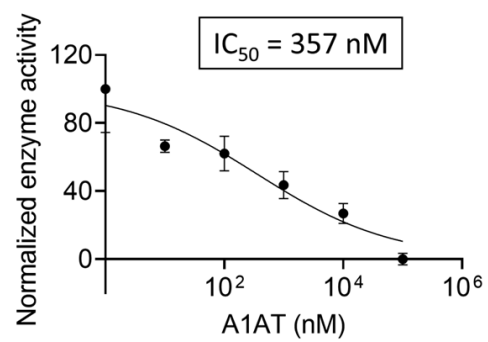

Figure 2. The effect of intracellular and extracellular inhibitors on TMPRSS2 activity.

Fluorescence of TMPRSS2-overexpressing cells was measured every 15 minutes in the presence of the indicated concentrations of camostat mesylate (camostat) (A), secretory leukocyte protease inhibitor (SLPI) (B), or A1AT (C). The results in A-C are presented as the means \pm SE from at least 3 independent experiments performed in duplicate and/or triplicate. TMPRSS2, transmembrane serine protease 2 .

\section{Analyzing the A1AT effect on TMPRSS2-mediated SARS-CoV-2 infectivity}

We investigated whether A1AT can inhibit SARS-CoV-2 infectivity in cells that are commonly used for SARS-CoV-2 assays because of their TMPRSS2 expression [1]. Caco-2 cells were either left untreated or treated with either A1AT $(10 \mu \mathrm{M})$ or camostat $(10 \mu \mathrm{M})$; cells were then infected with SARS-CoV-2. Twenty hours later, quantifying the genomic SARS-CoV-2 from the intracellular RNA revealed a significant decrease in the viral load in cells that were treated with A1AT or camostat (-2.6 and -3.9 fold, respectively) compared with untreated control cells $(P<0.0001$ for both inhibitors; Figure 4A). We then tested the effect of A1AT and camostat on the infectivity of SARS-CoV-2 in Calu-3 cells. Both inhibitors decreased SARS-CoV-2 plaque formation when analyzed 24 hours after infection (Figure 4B). A1AT did not decrease plaque formation 48 hours after infection, whereas the effect of camostat persisted at that time point (Figure 4B). These data suggest that A1AT can limit the SARS-CoV-2 life cycle by modulating TMPRSS2 activity in the host cells. Importantly, A1AT and camostat were nontoxic in Caco-2 cells in the tested concentrations (Supplementary Figure 3). 
A TMPRSS2
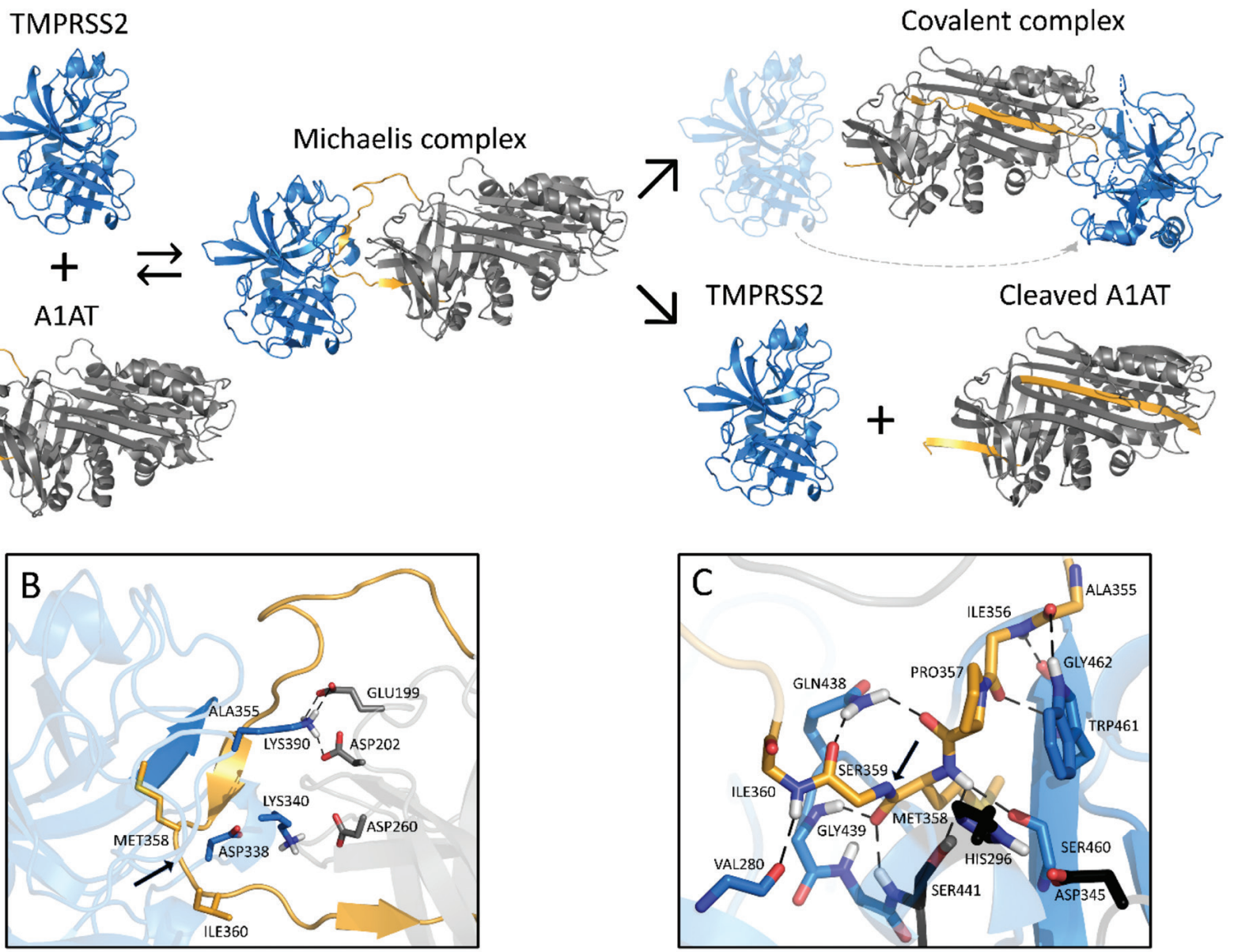

Figure 3. Schematic inhibition of TMPRSS2 by A1AT.

A. General inhibitory mechanism of serpins applied to TMPRSS2 and A1AT are shown. The homology model of TMPRSS2 is shown as the blue cartoon and A1AT as the grey cartoon, with the reactive center loop highlighted in gold. B. Shown are the interactions at the interface of the Michaelis complex model, highlighting LYS340 and LYS390 of TMPRSS2 (blue) and GLU199, ASP202, and ASP260 of A1AT (grey). C. A close-up of the Michaelis complex at the active site region is shown. The catalytic triad residues HIS296, ASP345, and SER441 are depicted in black. Relevant residues are represented as sticks, hydrogen bonds are represented as dashed black lines, and the cleavage site is indicated by a black arrow. Note that there are hydrogen bonds at the oxyanion hole between GLY439/SER441 of TMPRSS2 and MET358 of A1AT. A1AT, alpha 1 antitrypsin; TMPRSS2, transmembrane serine protease 2. 
A

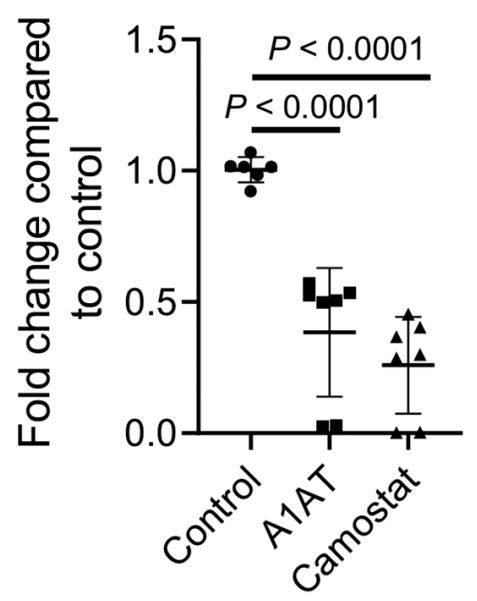

B

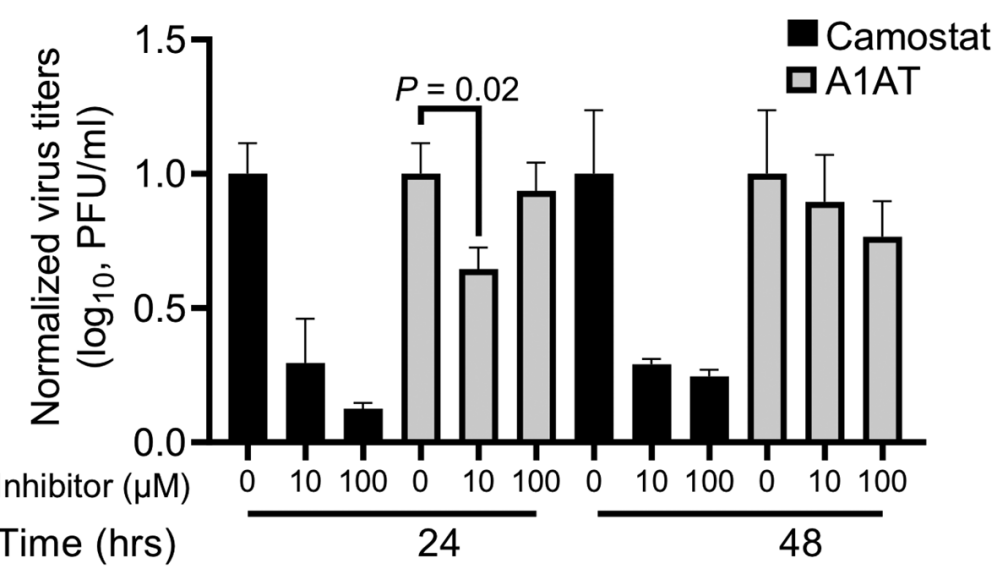

Figure 4. The effect of A1AT and camostat on SARS-CoV-2 infection.

A. Intracellular SARS-CoV-2 genomic copies were analyzed twenty hours after infection of Caco-2 cells in the presence of A1AT $(10 \mu \mathrm{M})$, camostat $(10 \mu \mathrm{M})$, or control media. Data represent fold change in $\mathrm{CoV}-2$ copy number compared to control media. Each data point represents 1 well with the mean $\pm \mathrm{SD}$ of 3 independent experiments. B. SARS-CoV-2 virus production was analyzed 24 and 48 hours after infection of Calu- 3 cells at the indicated concentration of A1AT and camostat and in control media. Data represent fold change in plaque number compared to control media. Results are the mean \pm SE of 2 or more independent experiments. A1 AT, alpha 1 antitrypsin; PFU, plaque-forming units.

\section{Plasma A1AT levels in patients with COVID-19}

$\mathrm{A} 1 \mathrm{AT}$ is normally found at high concentrations in the blood and increases during acute phase responses or tissue injury [14]. We hypothesized that A1AT concentrations in plasma samples from patients with COVID-19 would correlate with disease severity as part of the anti-SARSCoV-2 response. To test this hypothesis, we analyzed plasma A1AT levels in a cohort of patients who tested positive for COVID-19. These patients were divided into 3 groups according to disease severity at the time of emergency department disposition and the maximal severity within 30 days ( 1 mild - outpatient care, 2 moderate - need for hospitalization, 3 severe - need for intensive care unit admission; see methods section). A1AT levels were significantly different between the group of patients with mild disease and the group of patients with moderate disease. The mean concentration of A1AT was the highest in the group of patients with severe disease compared to the other groups (Figure 5A). A1AT concentrations positively correlated with maximal severity of disease (Figure 5B). Plasma A1AT concentrations correlated with plasma IL-6 $(\mathrm{r}=0.65, P<$ $0.0001)$, IL-10 $(\mathrm{r}=0.33, P=0.002)$, and TNFa concentrations $(\mathrm{r}=0.3 ; P=0.002)$ but not plasma IL-8 concentrations (Figure 5C,D). Consistent with previous studies [15], we observed that the IL6/A1AT ratio positively correlated with disease severity (Figure 5E,F) 
A

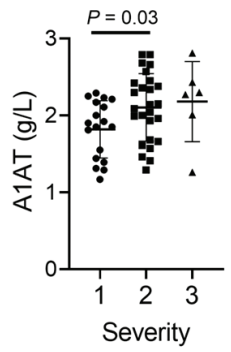

D

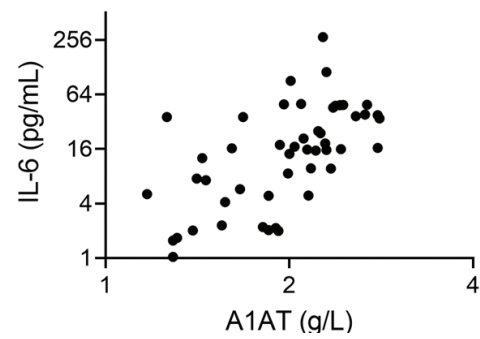

G

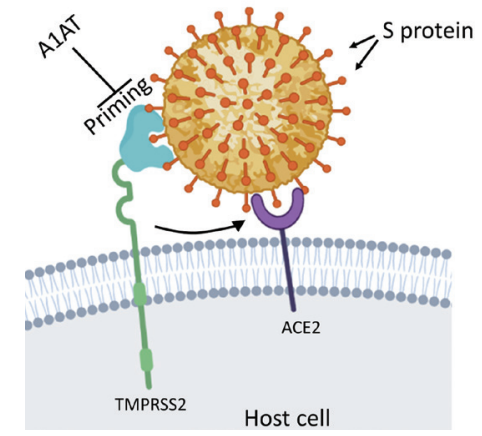

C

\begin{tabular}{|l|l|l|l|l|}
\hline & $\begin{array}{l}\text { A1AT vs. } \\
\text { IL-10 }\end{array}$ & $\begin{array}{l}\text { A1AT vs. } \\
\text { IL-6 }\end{array}$ & $\begin{array}{l}\text { A1AT vs. } \\
\text { IL-8 }\end{array}$ & $\begin{array}{l}\text { A1AT vs. } \\
\text { TNFa }\end{array}$ \\
\hline Spearman $r$ & 0.33 & 0.65 & 0.23 & 0.3 \\
\hline P value & 0.002 & $<0.0001$ & 0.14 & 0.04
\end{tabular}

E

$\mathbf{F}$

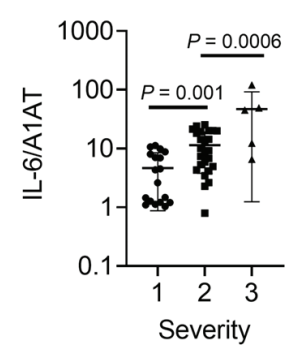

Figure 5. A1AT concentration in plasma samples of patients with COVID-19 and suggested role in SARS-CoV-2 cell entry.

A1AT plasma concentrations in patients who were positive for COVID-19 are shown and stratified according to disease severity at the time of disposition from the Emergency Department (A) or the maximal severity within 30 days of index Emergency Department visit (Max severity) (B) ( 1 = Outpatient, 2 $=$ Hospitalized, 3 = Intensive Care Unit or Death). In (C), correlation between plasma A1AT concentrations and plasma IL-10, IL-6, IL-8, or TNFa concentrations, $r$ and $P$ values were calculated according to Spearman correlation. D. Plasma concentration of A1AT and IL-6 in each patient with confirmed COVID-19 with markers representing individual patients. Ratio of IL-6/A1AT plasma concentrations in patients who were positive for COVID-19 are shown and stratified according to disease severity at the time of disposition from the Emergency Department (E) or the maximal severity within 30 days of index Emergency Department visit (Max severity) (F). G. Model of SARS-CoV-2 entry mediated by extracellular proteolytic events. Extracellular proteases, such as TMPRSS2, process the S protein on the SARS-CoV-2 envelope in a process called priming. Priming of the $S$ protein is necessary for binding between the $S$ protein and the host receptor angiotensin-converting enzyme 2 (ACE2). Extracellular inhibitors, such as A1AT, prevent the priming of the S protein and inhibit virus entry. In addition, inhibiting transmembrane serine protease 2 (TMPRSS2) prevents processing of ACE2, which decreases the infectivity of the coronavirus. Model was illustrated using Biorender. A1AT, alpha 1 antitrypsin; IL, interleukin 


\section{DISCUSSION}

Herein, we developed a cell-based methodology that allows the quantification of TMPRSS2 activity. This methodology enables testing the effect of intracellular compounds and extracellular compounds, thus permitting differentiation between inhibition of intracellular and extracellular protease. Using this methodology, we revealed that A1AT, which is approved by the FDA for the treatment of A1AT deficiency, can efficiently inhibit TMPRSS2. Structural modeling of the A1AT-TMPRSS2 Michaelis complex revealed that A1AT is likely cleaved and covalently bound to TMPRSS2. The importance of A1AT in fighting coronavirus infection was supported by the finding that plasma A1AT levels correlated with COVID-19 severity and with plasma IL-6 levels. A1AT inhibited SARS-CoV-2 infection at a comparable level to camostat in Caco-2 and Calu-3 cells, cell types that are efficiently infected by SARS-CoV-2 [16]. Consistent with our findings, Oguntuyo et al demonstrated that SARS-CoV-2 naive serum exhibits significant inhibition of SARS-CoV-2 entry and that this inhibition is mostly accounted for by the presence of A1AT in the sera [17]. Therefore, these collective data suggest that A1AT treatment may benefit COVID-19 countermeasures by inhibiting extracellular-mediated S protein processing and virus entry. Notably, the inhibitory effect of camostat on SARS-CoV-2 infection in Calu-3 cells persisted longer than that of A1AT, which inhibited the infection after 24 hours but not after 48 hours. This difference may stem from a different mechanism of action of these 2 drugs; A1AT inhibits extracellular and membranal proteases, whereas camostat penetrates the cells. Therefore, camostat can potentially inhibit intracellular proteases that affect multiple steps in the SARS-CoV-2 life cycle, which therefore prolongs its effect.

Though the relative contribution of intracellular proteases and extracellular proteases to the $S$ protein priming and cell entry of SARS-CoV-2 has yet to be determined, we provide evidence that extracellular protease activity is rate-limiting in the process of SARS-CoV-2 cell entry. Therefore, we suggest that extracellular protease inhibitor delivery may provide a good strategy for inhibiting SARS-CoV-2 entry and cell-to-cell transmission by modulating the exterior of the host cells. The disappointing results of hydroxychloroquine, which interferes with the activity of intracellu-

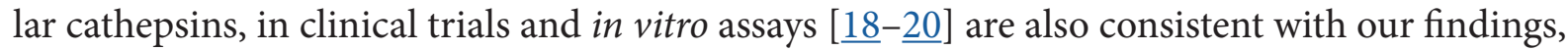
which support the importance of extracellular proteases. Notably, neither A1AT nor camostat completely blocked SARS-CoV-2 entry. This could be explained by slow, unprocessed ACE2-mediated cell entry in the absence of TMPRSS2 and/or expression of other proteases, including intracellular proteases that may cleave the $\mathrm{S}$ protein.

Targeting the host extracellular proteases, such as TMPRSS2, has several advantages over targeting viral proteins. First, anti-virals can rapidly lose their effectiveness due to the high rate of mutations that occur in the viral genome, but targeting host proteins limits the risk of drug-resistant viruses due to the relatively low rate of mutations in the host genome. Notably, the obstacle in targeting human proteins is the potential risk of altering physiologic pathways. It has been suggested that TMPRSS2 initiates a cascade of proteolytic activation events that regulate processing of proteins in seminal fluid and in the lung because TMPRSS2 regulates the sodium channel ENaC [21]. Nevertheless, mice deficient in TMPRSS2 lack any obvious phenotypes, suggesting that other proteases may have redundant roles and may compensate for the loss of TMPRSS2 [22]. Therefore, delivery of TMPRSS2 inhibitors during viral infections is likely a relatively safe strategy. Although the safety of TMPRSS2 inhibition has not been clinically proven yet, drugs with proteolytic inhibition activity towards TMPRSS2 (eg, camostat mesylate and nafamostat mesylate) are currently being 
pursued for the treatment of COVID-19 [르-27]. Notably, unlike extracellular A1AT, camostat and nafamostat are cell permeable and therefore may possess undesired intracellular protease inhibition capacity. Second, inhibiting cell entry is an upstream intervention method that limits the overall viral burden and the spread to and replication within tissues, such as the salivary glands, that have important pathologic consequences involved in viral transmission to others [28]. Third, inhibiting cell entry may prevent several downstream disease outcomes. For example, SARS-CoV-2-infected cells undergo cell death by pyroptosis, a process that is thought to induce complications, such as cytokine storm and intra-vascular thrombosis, and thereby results in severe disease outcomes [29]. Moreover, cell death of key alveolar cells induces tissue damage that progresses into acute respiratory distress syndrome, a severe clinical phenotype of COVID-19 [이] Therefore, inhibiting SARS-CoV-2 infection and cell entry by inhibiting the proteolytic priming of the coronavirus S protein is likely to decrease cell death and thereby reduce disease severity.

To our knowledge, we are the first to demonstrate that A1AT inhibits TMPRSS2, which is an extracellular protease with a key role in the entry of SARS-CoV-2, SARS-CoV, MERS-CoV, and influenza viruses $[\underline{1}, \underline{31}-\underline{35}]$. A1AT belongs to the super family of serine protease inhibitors (SERPIN) that irreversibly inhibit serine and cysteine proteases. Proteases interact with SERPINs as depicted in Figure 3A, forming a Michaelis complex in which the reactive center loop (RCL) binds the protease active site (modelled for TMPRSS2 and A1AT in this work). Cleavage of the RCL results in the formation of a transient, covalent complex that can either undergo dissociation (lower right pathway in Figure 3A; cleaved A1AT represented by PDB 7API [ [36]) or translocation and irreversible inhibition [글 (upper right pathway in Figure 3A; represented by PDB 2D26 [38] in the absence of a TMPRSS2-A1AT-specific model). One of the fragments of the cleaved RCL is inserted into the central beta-sheet of A1AT in both pathways, whereas the other fragment (36aa) is released into the solvent. Notably, the 36-aa fragment is produced as a result of proteolytic cleavage by several serine proteases and possesses physiologic functions [ $\underline{39}-\underline{41}$ ]. The experiments with virus infection in vitro provide a proof of principle that A1AT not only biochemically inhibits TMPRSS2 but also possesses the ability to inhibit SARS-CoV-2 infection. It could be argued that we only found a modest reduction in SARS-CoV-2 infection of Calu-3 and Caco-2 cells, but it is important that the test compound (A1AT) was as effective as the positive control camostat in the early time point. It is notable that both agents are now in clinical trials for COVID-19 so the true effectiveness in patients with COVID-19 will be determined.

If endogenous A1AT was solely anti-inflammatory, one may have expected a negative correlation between sera levels of A1AT and COVID-19 severity. However, endogenous A1AT concentration in the blood can be increased by 6 -fold as part of the acute phase of inflammation or tissue injury [14]. A1AT is known to inhibit neutrophil elastase, proteinase 3, and cathepsin G. Neutrophil elastase cleaves several structural proteins in the lungs, processes several innate immune mediators, and has been shown to be involved in the pathogenicity of SARS-CoV-2 substrains by cleaving the S1-S2 junction of the S protein [르. In addition, A1AT promotes clearance of apoptotic cells [푹. Increased neutrophil levels have been found in patients with COVID-19 with severe

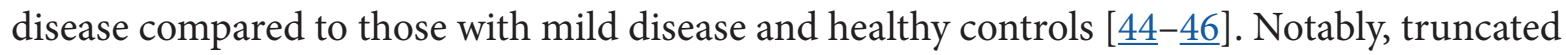
forms of A1AT were significantly increased in the serum of patients with SARS compared to control patients, and the truncated A1AT levels correlated with disease severity [47]. These findings suggest that A1AT may be a part of a natural protective mechanism to fight coronavirus infection and acute lung disease. Indeed, we demonstrated that plasma A1AT levels were associated with 
COVID-19 severity and with IL-6, a cytokine that has been implicated in COVID-19 pathology and as a biomarker for disease severity $[\underline{15}, \underline{46}, \underline{48}-\underline{50}]$. A high ratio of IL-6/A1AT was associated with more severe disease outcome, consistent with a recent report [15]. Notably, A1AT was shown to inhibit the infection of $\mathrm{H} 3 \mathrm{~N} 2$ influenza A and influenza B viruses in a murine model [ $\underline{31}$ ], even though these viruses do not require TMPRSS2 priming, underscoring that A1AT can mediate anti-viral effects via multiple mechanisms. Finally, though endogenous A1AT positively correlated with COVID-19 severity, it is notable that our findings represent potential therapeutic effects of exogenously administered A1AT.

We suggest that treatment with extracellular protease inhibitors either alone or in combination with other anti-COVID-19 agents may be a useful antiviral strategy to fight COVID-19. These protease inhibitors have the potential to prevent SARS-CoV-2 entry to host cells by inhibiting $S$ protein priming by TMPRSS2 and other extracellular proteases and binding of the virus to ACE2 (Figure 5G). A1AT may be particularly effective as it has dual capacity, inhibiting TMPRSS2 (and hence viral uptake and subsequent replication) and possessing anti-inflammatory activity [51]. We suggest that using these inhibitors may be therapeutic in conditions in which TMPRSS2 function is pathogenic, such as in several types of coronavirus and influenza infections. The ready availability of and safety profile of A1AT calls attention to its potential clinical use for the COVID-19 pandemic.

\section{AUTHOR CONTRIBUTIONS}

N.P.A. designed and performed experiments and data analysis and wrote the paper, A.M.K. performed experiments, and V.C. and I.V.A. performed experiments with SARS-CoV-2. K.E. and L.R. assisted in data generation and analysis of template-based molecular docking. B.M.H., J.L.B., and S.W.B. conducted patient sample collection and plasma tests of patients with COVID-19. F.N. supervised the template-based molecular docking. K.K-H. supervised the SARS-CoV-2 experiments. M.E.R. supervised the study.

\section{POTENTIAL CONFLICTS OF INTEREST}

M.E.R. is a consultant for Pulm One, Spoon Guru, ClostraBio, Serpin Pharma, Celgene, Astra Zeneca, Allakos, Arena Pharmaceuticals, Guidepoint, and Suvretta Capital Management and has an equity interest in the first 4 listed and royalties from reslizumab (Teva Pharmaceuticals), PEESSv2 (Mapi Research Trust), and UpToDate. M.E.R. is an inventor of patents owned by Cincinnati Children's Hospital. M.E.R. and N.P.A. are inventors of a patent owned by Cincinnati Children's Hospital with the provisional number of 63/017,027.

\section{SUPPLEMENTARY DATA}

Supplementary materials are available at the Pathogens and Immunity website. Supplementary data may be provided by the authors to benefit the reader. Supplementary data are not copyedited and are the sole responsibility of the authors. Questions or comments related to supplementary materials should be addressed to the corresponding author.

Supplementary Figure 1

Supplementary Figure 2

Supplementary Figure 3 


\section{ACKNOWLEDGMENTS}

We thank M. Brantly (Alpha-1 Foundation) for the gift of A1AT and B.J. Aronow, J.D. Molkentin, and S.N. Waggoner (Cincinnati Children's Hospital) for insightful discussion and advice. We also thank S. Hottinger (Cincinnati Children's Hospital) for editorial assistance. The following reagent was deposited by the Centers for Disease Control and Prevention and obtained through BEI Resources, NIAID, NIH: SARS-Related Coronavirus 2 Isolate USA-WA1/2020, NR-52281.

\section{FUNDING}

This work was supported in part by NIH R37 AI045898, U19 AI070235, R01 AI057803, R01 DK107502, and P30 DK078392 (Gene and Protein Expression Core); the Campaign Urging Research for Eosinophilic Disease (CURED); the University of Cincinnati College of Medicine Special Coronavirus (COVID-19) Research Pilot Grant Program; and the Sunshine Charitable Foundation and its supporters, Denise and David Bunning (to M.E.R.). This work was also supported in part by the European Commission (ERC CoG 772230), MATH+: The Berlin Mathematics Research Center, AA1-6, Deutsche Forschungsgemeinschaft (SFB1114/C03).

\section{REFERENCES}

1. Hoffmann M, Kleine-Weber H, Schroeder S, Kruger N, Herrler T, Erichsen S, Schiergens TS, Herrler G, Wu NH, Nitsche A, Muller MA, Drosten C, Pohlmann S. SARSCoV-2 cell entry depends on ACE2 and TMPRSS2 and is blocked by a clinically proven protease inhibitor. Cell. 2020;181(2):271-80 e8. doi: 10.1016/j.cell.2020.02.052. PubMed PMID: 32142651; PMCID: PMC7102627.

2. Qing E, Hantak MP, Galpalli GG, Gallagher T. Evaluating MERS-CoV entry pathways. Methods Mol Biol. 2020;2099:9-20. doi: 10.1007/978-1-0716-0211-9 2. PubMed PMID: 31883084; PMCID: PMC7121971.

3. Heurich A, Hofmann-Winkler H, Gierer S, Liepold T, Jahn O, Pohlmann S. TMPRSS2 and ADAM17 cleave ACE2 differentially and only proteolysis by TMPRSS2 augments entry driven by the severe acute respiratory syndrome coronavirus spike protein. J Virol. 2014;88(2):1293-307. doi: 10.1128/JVI.02202-13. PubMed PMID: 24227843; PMCID: PMC3911672.

4. Iwata-Yoshikawa N, Okamura T, Shimizu Y, Hasegawa H, Takeda M, Nagata N. TMPRSS2 Contributes to virus spread and immunopathology in the airways of murine models after coronavirus infection. J Virol. 2019;93(6). doi: 10.1128/JVI.01815-18. PubMed PMID: 30626688; PMCID: PMC6401451.

5. Matsuyama S, Nao N, Shirato K, Kawase M, Saito S, Takayama I, Nagata N, Sekizuka T, Katoh H, Kato F, Sakata M, Tahara M, Kutsuna S, Ohmagari N, Kuroda M, Suzuki T, Kageyama T, Takeda M. Enhanced isolation of SARS-CoV-2 by TMPRSS2-expressing cells. Proc Natl Acad Sci U S A. 2020;117(13):7001-3. doi: 10.1073/pnas.2002589117. PubMed PMID: 32165541; PMCID: PMC7132130.

6. Reihill JA, Walker B, Hamilton RA, Ferguson TE, Elborn JS, Stutts MJ, Harvey BJ, Saint-Criq V, Hendrick SM, Martin SL. Inhibition of protease-epithelial sodium channel signaling improves mucociliary function in cystic fibrosis airways. Am J Respir Crit Care Med. 2016;194(6):701-10. doi: 10.1164/rccm.201511-2216OC. PubMed 
PMID: 27014936.

7. Strnad P, McElvaney NG, Lomas DA. Alpha1-antitrypsin deficiency. N Engl J Med. 2020;382(15):1443-55. doi: 10.1056/NEJMra1910234. PubMed PMID: 32268028.

8. Baer A, Kehn-Hall K. Viral concentration determination through plaque assays: Using traditional and novel overlay systems. J Vis Exp. 2014(93):e52065. doi: 10.3791/52065. PubMed PMID: 25407402; PMCID: PMC4255882.

9. Dominguez C, Boelens R, Bonvin AM. HADDOCK: A protein-protein docking approach based on biochemical or biophysical information. J Am Chem Soc. 2003;125(7):1731-7. doi: 10.1021/ja026939x. PubMed PMID: 12580598.

10. Dementiev A, Simonovic M, Volz K, Gettins PG. Canonical inhibitor-like interactions explain reactivity of alpha1-proteinase inhibitor pittsburgh and antithrombin with proteinases. J Biol Chem. 2003;278(39):37881-7. doi: 10.1074/jbc.M305195200. PubMed PMID: 12860985.

11. Rensi S, Keys A, Y-C, Derry A, McInnes G, Liu T, Altman R. Homology modeling of TMPRSS2 yields candidate drugs that may inhibit entry of SARS-CoV-2 into human cells. ChemRxiv. 2020. doi: 10.26434/chemrxiv.12009582.v1. PMID: 32511288.

12. Xue LC, Dobbs D, Honavar V. HomPPI: A class of sequence homology based protein-protein interface prediction methods. BMC Bioinformatics. 2011;12:244. doi: 10.1186/1471-2105-12-244. PubMed PMID: 21682895 ; PMCID: PMC3213298.

13. Tim Hempel LR, Simon Olsson, Nurit P. Azouz, Andrea M. Klingler, Marc E. Rothenberg, Frank Noé. Molecular mechanism of SARS-CoV-2 cell entry inhibition via TMPRSS2 by camostat and nafamostat mesylate. bioRxiv2020.

14. Guttman O, Baranovski BM, Schuster R, Kaner Z, Freixo-Lima GS, Bahar N, Kalay N, Mizrahi MI, Brami I, Ochayon DE, Lewis EC. Acute-phase protein alpha1-anti-trypsin: Diverting injurious innate and adaptive immune responses from non-authentic threats. Clin Exp Immunol. 2015;179(2):161-72. doi: 10.1111/cei.12476. PubMed PMID: 25351931; PMCID: PMC4298394.

15. McElvaney OJ, McEvoy NL, McElvaney OF, Carroll TP, Murphy MP, Dunlea DM, Ni Choileain O, Clarke J, O’Connor E, Hogan G, Ryan D, Sulaiman I, Gunaratnam C, Branagan P, O’Brien ME, Morgan RK, Costello RW, Hurley K, Walsh S, de Barra E, McNally C, McConkey S, Boland F, Galvin S, Kiernan F, O’Rourke J, Dwyer R, Power M, Geoghegan P, Larkin C, O’Leary RA, Freeman J, Gaffney A, Marsh B, Curley GF, McElvaney NG. Characterization of the inflammatory response to severe COVID-19 illness. Am J Respir Crit Care Med. 2020;202(6):812-21. doi: 10.1164/rccm.202005-1583OC. PubMed PMID: 32584597; PMCID: PMC7491404.

16. Chu H, Chan JF, Yuen TT, Shuai H, Yuan S, Wang Y, Hu B, Yip CC, Tsang JO, Huang X, Chai Y, Yang D, Hou Y, Chik KK, Zhang X, Fung AY, Tsoi HW, Cai JP, Chan WM, Ip JD, Chu AW, Zhou J, Lung DC, Kok KH, To KK, Tsang OT, Chan KH, Yuen KY. Comparative tropism, replication kinetics, and cell damage profiling of SARSCoV-2 and SARS-CoV with implications for clinical manifestations, transmissibility, and laboratory studies of COVID-19: An observational study. Lancet Microbe. 2020;1(1):e14-e23. doi: 10.1016/S2666-5247(20)30004-5. PubMed PMID: 32835326; 
PMCID: PMC7173822.

17. Oguntuyo KY, Stevens CS, Siddiquey MN, Schilke RM, Woolard MD, Zhang H, Acklin JA, Ikegame S, Hung CT, Lim JK, Cross RW, Geisbert TW, Ivanov SS, Kamil JP, Lee B. In plain sight: The role of alpha-1-antitrypsin in COVID-19 pathogenesis and therapeutics. bioRxiv. 2020. doi: 10.1101/2020.08.14.248880. PubMed PMID: 32817940; PMCID: PMC7430570.

18. Geleris J, Sun Y, Platt J, Zucker J, Baldwin M, Hripcsak G, Labella A, Manson DK, Kubin C, Barr RG, Sobieszczyk ME, Schluger NW. Observational study of hydroxychloroquine in hospitalized patients with COVID-19. N Engl J Med. 2020;382(25):2411-8. doi: 10.1056/NEJMoa2012410. PubMed PMID: 32379955; PMCID: PMC7224609.

19. Kalligeros M, Shehadeh F, Atalla E, Mylona EK, Aung S, Pandita A, Larkin J, Sanchez M, Touzard-Romo F, Brotherton A, Shah R, Cunha CB, Mylonakis E. Hydroxychloroquine use in hospitalized patients with COVID-19: An observational matched cohort study. J Glob Antimicrob Resist. 2020. doi: 10.1016/j.jgar.2020.07.018. PubMed PMID: 32763357; PMCID: PMC7403006.

20. Tianling Ou HM, Lizhou Zhang, Amrita Ojha, Hyeryun Choe, Michael Farzan. Hydroxychloroquine-mediated inhibition of SARS-CoV-2 entry is attenuated by TMPRSS2. BioRxiv2020.

21. Donaldson SH, Hirsh A, Li DC, Holloway G, Chao J, Boucher RC, Gabriel SE. Regulation of the epithelial sodium channel by serine proteases in human airways. J Biol Chem. 2002;277(10):8338-45. doi: 10.1074/jbc.M105044200. PubMed PMID: 11756432.

22. Kim TS, Heinlein C, Hackman RC, Nelson PS. Phenotypic analysis of mice lacking the Tmprss2-encoded protease. Mol Cell Biol. 2006;26(3):965-75. doi: 10.1128/ MCB.26.3.965-975.2006. PubMed PMID: 16428450; PMCID: PMC1347042.

23. Camostat Mesylate in COVID-19 Outpatients. ClinicalTrials.gov identifier: NCT04353284. Posted April 20, 2020. Updated November 24, 2020. https://clinicaltrials.gov/ct2/show/NCT04353284?term=camostat\&cond=Covid-19\&draw=2\&rank=1.

24. The Utility of Camostat Mesylate in Patients With COVID-19 Associated Coagulopathy (CAC) and Cardiovascular Compli. ClinicalTrials.gov identifier: NCT04435015. Posted June 17, 2020. Updated October 28, 2020. https://clinicaltrials.gov/ct2/show/ NCT04435015?term = camostat\&cond=Covid-19\&draw=2\&rank=2.

25. Clinical Efficacy of Nafamostat Mesylate for COVID-19 Pneumonia. ClinicalTrials.gov identifier: NCT04418128. Posted June 5, 2020. Updated June 9, 2020. https://clinicaltrials.gov/ct2/show/NCT04418128?term=nafamostat\&cond=Covid-19\&draw=2.

26. Study to Evaluate the Safety and Efficacy of Liquid Alpha1-Proteinase Inhibitor (Human) in Hospitalized Participants With Coronavirus Disease (COVID-19) 2020. ClinicalTrials.gov identifier: NCT04547140. Posted September 4, 2020. Updated March 1, 2021. https://clinicaltrials.gov/ct2/show/NCT04547140?term=alpha+1\&cond=Covid19\&draw=2\&rank=1. 
27. Study to Evaluate the Safety and Efficacy of Prolastin in Hospitalized Subjects With COVID-19 2020. ClinicalTrials.gov identifier: NCT04495101. Posted July 31, 2020. Updated February 21, 2021. https://clinicaltrials.gov/ct2/show/NCT04495101?ter$\underline{\mathrm{m}=\text { alpha+1 \&cond }=\text { Covid19\&draw=2\&rank=6. }}$.

28. Song J, Li Y, Huang X, Chen Z, Li Y, Liu C, Chen Z, Duan X. Systematic analysis of ACE2 and TMPRSS2 expression in salivary glands reveals underlying transmission mechanism caused by SARS-CoV-2. J Med Virol. 2020;92(11):2556-66. doi: 10.1002/ jmv.26045. PubMed PMID: 32441816; PMCID: PMC7280739.

29. Yap JKY, Moriyama M, Iwasaki A. Inflammasomes and pyroptosis as therapeutic targets for COVID-19. J Immunol. 2020;205(2):307-12. doi: 10.4049/jimmunol.2000513. PubMed PMID: 32493814; PMCID: PMC7343621.

30. Chen J, Wu H, Yu Y, Tang N. Pulmonary alveolar regeneration in adult COVID-19 patients. Cell Res. 2020;30(8):708-10. doi: 10.1038/s41422-020-0369-7. PubMed PMID: 32632255; PMCID: PMC7338112.

31. Harbig A, Mernberger M, Bittel L, Pleschka S, Schughart K, Steinmetzer T, Stiewe T, Nist A, Bottcher-Friebertshauser E. Transcriptome profiling and protease inhibition experiments identify proteases that activate $\mathrm{H} 3 \mathrm{~N} 2$ influenza $\mathrm{A}$ and influenza $\mathrm{B}$ viruses in murine airways. J Biol Chem. 2020;295(33):11388-407. doi: 10.1074/jbc. RA120.012635. PubMed PMID: 32303635; PMCID: PMC7450141.

32. Gierer S, Bertram S, Kaup F, Wrensch F, Heurich A, Kramer-Kuhl A, Welsch K, Winkler M, Meyer B, Drosten C, Dittmer U, von Hahn T, Simmons G, Hofmann H, Pohlmann $\mathrm{S}$. The spike protein of the emerging betacoronavirus EMC uses a novel coronavirus receptor for entry, can be activated by TMPRSS2, and is targeted by neutralizing antibodies. J Virol. 2013;87(10):5502-11. doi: 10.1128/JVI.00128-13. PubMed PMID: 23468491 ; PMCID: PMC3648152.

33. Shirato K, Kawase M, Matsuyama S. Middle East respiratory syndrome coronavirus infection mediated by the transmembrane serine protease TMPRSS2. J Virol. 2013;87(23):12552-61. doi: 10.1128/JVI.01890-13. PubMed PMID: 24027332; PMCID: PMC3838146.

34. Hatesuer B, Bertram S, Mehnert N, Bahgat MM, Nelson PS, Pohlmann S, Schughart $\mathrm{K}$. Tmprss2 is essential for influenza H1N1 virus pathogenesis in mice. PLoS Pathog. 2013;9(12):e1003774. doi: 10.1371/journal.ppat.1003774. PubMed PMID: 24348248; PMCID: PMC3857797.

35. Sakai K, Ami Y, Tahara M, Kubota T, Anraku M, Abe M, Nakajima N, Sekizuka T, Shirato K, Suzaki Y, Ainai A, Nakatsu Y, Kanou K, Nakamura K, Suzuki T, Komase K, Nobusawa E, Maenaka K, Kuroda M, Hasegawa H, Kawaoka Y, Tashiro M, Takeda $\mathrm{M}$. The host protease TMPRSS2 plays a major role in in vivo replication of emerging H7N9 and seasonal influenza viruses. J Virol. 2014;88(10):5608-16. doi: $\underline{10.1128 /}$ JVI.03677-13. PubMed PMID: 24600012; PMCID: PMC4019123.

36. Engh R, Lobermann H, Schneider M, Wiegand G, Huber R, Laurell CB. The S variant of human alpha 1-antitrypsin, structure and implications for function and metabolism. Protein Eng. 1989;2(6):407-15. doi: 10.1093/protein/2.6.407. PubMed PMID: 
2785270.

37. Bao J, Pan G, Poncz M, Wei J, Ran M, Zhou Z. Serpin functions in host-pathogen interactions. PeerJ. 2018;6:e4557. doi: 10.7717/peerj.4557. PubMed PMID: 29632742; PMCID: PMC5889911.

38. Dementiev A, Dobo J, Gettins PG. Active site distortion is sufficient for proteinase inhibition by serpins: Structure of the covalent complex of alpha1-proteinase inhibitor with porcine pancreatic elastase. J Biol Chem. 2006;281(6):3452-7. doi: 10.1074/jbc. M510564200. PubMed PMID: 16321984.

39. Blaurock N, Schmerler D, Hunniger K, Kurzai O, Ludewig K, Baier M, Brunkhorst FM, Imhof D, Kiehntopf M. C-terminal alpha-1 antitrypsin peptide: A new sepsis biomarker with immunomodulatory function. Mediators Inflamm. 2016;2016:6129437. doi: 10.1155/2016/6129437. PubMed PMID: 27382189 ; PMCID: PMC4921625.

40. Risor MW, Juhl DW, Bjerring M, Mathiesen J, Enghild JJ, Nielsen NC, Otzen DE. Critical influence of cosolutes and surfaces on the assembly of serpin-derived amyloid fibrils. Biophys J. 2017;113(3):580-96. doi: 10.1016/j.bpj.2017.06.030. PubMed PMID: 28793213 ; PMCID: PMC5549687.

41. Potere N, Del Buono MG, Niccoli G, Crea F, Toldo S, Abbate A. Developing LRP1 agonists into a therapeutic strategy in acute myocardial infarction. Int J Mol Sci. 2019;20(3). doi: 10.3390/ijms20030544. PubMed PMID: 30696029; PMCID: PMC6387161.

42. Chandrika Bhattacharyya CD, Arnab Ghosh, Animesh K. Singh, Souvik Mukherjee, Partha P. Majumder, Analabha Basu, Nidhan K. Biswas. Global spread of SARSCoV-2 subtype with spike protein mutation D614G is shaped by human genomic variations that regulate expression of TMPRSS2 and MX1 genes. BioRxiv. 2020. doi: $\underline{10.1101 / 2020.05 .04 .075911}$

43. Serban KA, Petrusca DN, Mikosz A, Poirier C, Lockett AD, Saint L, Justice MJ, Twigg HL, 3rd, Campos MA, Petrache I. Alpha-1 antitrypsin supplementation improves alveolar macrophages efferocytosis and phagocytosis following cigarette smoke exposure. PLoS One. 2017;12(4):e0176073. doi: 10.1371/journal.pone.0176073. PubMed PMID: 28448535 ; PMCID: PMC5407578.

44. Qin C, Zhou L, Hu Z, Zhang S, Yang S, Tao Y, Xie C, Ma K, Shang K, Wang W, Tian DS. Dysregulation of immune response in patients with coronavirus 2019 (COVID-19) in Wuhan, China. Clin Infect Dis. 2020;71(15):762-8. doi: 10.1093/cid/ ciaa248. PubMed PMID: 32161940; PMCID: PMC7108125.

45. Wang D, Hu B, Hu C, Zhu F, Liu X, Zhang J, Wang B, Xiang H, Cheng Z, Xiong Y, Zhao Y, Li Y, Wang X, Peng Z. Clinical characteristics of 138 hospitalized patients with 2019 novel coronavirus-infected pneumonia in Wuhan, China. JAMA. 2020;323(11):1061-9. doi: 10.1001/jama.2020.1585. PubMed PMID: 32031570; PMCID: PMC7042881.

46. Henry BM, de Oliveira MHS, Benoit S, Plebani M, Lippi G. Hematologic, biochemical and immune biomarker abnormalities associated with severe illness and mortality in coronavirus disease 2019 (COVID-19): A meta-analysis. Clin Chem Lab Med. 
2020;58(7):1021-8. doi: 10.1515/cclm-2020-0369. PubMed PMID: 32286245.

47. Ren Y, He QY, Fan J, Jones B, Zhou Y, Xie Y, Cheung CY, Wu A, Chiu JF, Peiris JS, Tam $\mathrm{PK}$. The use of proteomics in the ciscovery of serum biomarkers from patients with severe acute respiratory syndrome. Proteomics. 2004;4(11):3477-84. doi: 10.1002/ pmic.200400897. PubMed PMID: 15378763; PMCID: PMC7167722.

48. Chen X, Zhao B, Qu Y, Chen Y, Xiong J, Feng Y, Men D, Huang Q, Liu Y, Yang B, Ding J, Li F. Detectable serum SARS-CoV-2 viral load (RNAaemia) is closely correlated with drastically elevated interleukin 6 (IL-6) level in critically ill COVID-19 patients. Clin Infect Dis. 2020. doi: 10.1093/cid/ciaa449. PubMed PMID: 32301997; PMCID: PMC7184354.

49. Ulhaq ZS, Soraya GV. Interleukin-6 as a potential biomarker of COVID-19 progression. Med Mal Infect. 2020;50(4):382-3. doi: 10.1016/j.medmal.2020.04.002. PubMed PMID: 32259560; PMCID: PMC7129451.

50. Zhou F, Yu T, Du R, Fan G, Liu Y, Liu Z, Xiang J, Wang Y, Song B, Gu X, Guan L, Wei Y, Li H, Wu X, Xu J, Tu S, Zhang Y, Chen H, Cao B. Clinical course and risk factors for mortality of adult inpatients with COVID-19 in Wuhan, China: A retrospective cohort study. Lancet. 2020;395(10229):1054-62. doi: 10.1016/S0140-6736(20)30566-3. PubMed PMID: 32171076; PMCID: PMC7270627.

51. Janciauskiene $S$, Welte T. Well-known and less well-known functions of alpha-1 antitrypsin. Its role in chronic obstructive pulmonary disease and other disease developments. Ann Am Thorac Soc. 2016;13 Suppl 4:S280-8. doi: 10.1513/AnnalsATS.201507-468KV. PubMed PMID: 27564662.

\section{FOOTNOTES}

Submitted November 27, 2020 | March 12, 2021 | Published April 26, 2021

\section{COPYRIGHT}

Copyright (C) 2021 Pathogens and Immunity. This is an open-access article distributed under the terms of the Creative Commons Attribution 4.0 International License. 